\title{
A FEASIBILITY STUDY FOR ENERGY AUTONOMY IN MULTI ROBOT SEARCH AND RESCUE OPERATIONS
}

\author{
Yosoph Sindi, Tony Pipe, Sanja Dogramadzi, Alan Winfeild, Chris Melhuish \\ Bristol Robotics Laboratory, University of the west of England, Coldharbour Lane, \\ Bristol BS16 1QY, United Kingdom.
}

\begin{abstract}
This paper proposes a novel search and rescue concept that aims to overcome the most basic obstacle in utilising a search and rescue teleoperated robot for a long distance energy autonomy. The concept utilizes a number of small robots capable of creating an energy supply chain that extends, to a degree based on the requirements of the search area. In this present work, the collaborative group of robots' predominant task is to maintain a constant supply of energy to the leading robot. Feasibility of the energy transfer and 'energy cost' have been simulated which consequently produced a mathematical description of the cost function. The results presented are obtained using a set of identical robots capable of conveying energy from the last deployed all the way to the leading robot. A robot single-line formation is important as it maps out the most frugal energy supply line. The methodology used was to simulate both methods of energy transfer, robots charging each other and robots exchanging batteries. The first method was implemented using MARCO2 (an in house built robot) to test the validity of the computer simulation and to study the effect of inaccurate localisation on the system.
\end{abstract}

\section{Introduction:}

Robots are well suited to address the limitations of Urban Search and Rescue. Much research has been invested in designing a suitable robot from an actuation perspective (Tsukagoshi 2005)[1], sensor fusion (Burke 2004)[2]. Most are mainly focused on either tethered or autonomous. Tethered robots are seriously restricted on scope and usability due to many sinuous pathways in a typical search and rescue environment. A non-tethered robot is governed by the fuel cell life (batteries in most cases). There are many aspects to be tackled but the scope of this project is to study the feasibility of a teleoperated rescue-robot and semi-autonomous followers able to transmit data wirelessly from the base and convey energy to the leader, to reach the furthest distance possible.

\subsection{Key words:}

Exergy, coefficient of restitution, Slack-line, Stride, trophallaxis, travelling salesman and Myrmecocystus melliger. 


\section{Literature review:}

In 2000 Rybski et al [3] created a swarm of robots and a leader for surveillance purposes. They had the ability to explore, map, locate and perform heterogeneous swarm operations. P.Basu (2004) [4] has proposed that the platoon should maintain communication bridges via its formation or distance between nodes should be reduced so that each node is connected to at least two nodes. J.Huang. et al (2006) [5] have introduced a 'follow the leader' system of automated highway barrel robots, where localization was done by GPS, deadreckoning and a laser range-finder. In collapsed buildings, a GPS signal is unreliable; nevertheless robots that monitor each other's location can be implemented. J.Huang et al [5] has also shown that a dead reckoning system for followers will contain a high degree of error. Dung et al (2006) [6] created sociable robots with a battery exchanging mechanism. Lits et al (2007) [7] also tackled the problem of distributing energy. The latter two had algorithms similar to the travelling salesman problem. Kub \& Melhuish (2004) [8] investigated robots managing their energy and trophallaxis. These robots used microbial fuel cells and were able to collect food from the environment. Their task was to reach the furthest distance possible. Finally K. Dumpert talks about these methods of energy exchange and storage in his book the social biology of ants (1981) [9].

\section{Energy transmission}

Example (1): If there is an infinite line of people, passing a glass of water; How far along the line would we be able to have a glass with some water in it? Case (A): If each person drinks a fixed amount of that water, the glass of water will reach the furthest distance if losses would be as minimal as possible.

Case (B): If once the glass is half empty they stop passing it and wait for a second glass to arrive in order to refill it then carry on passing the first, and so on. This is similar to A but allows the water to travel further. However, what is the furthest achievable distance?

This is also similar to the 'snooker balls' collision kinematics but with greater energy losses. For a platoon of robots each robot has to charge the preceding robot. Because robots do not join in mass after collision, the system cannot be modeled as a perfect inelastic collision. Velocity and mass are dynamic factors, they can be replaced by a symbol of energy regardless of the method by which energy (or Exergy) enters or exits the system. Using the coefficient of restitution, we can represent each of the robots' energy exchange by the following equation (1). 


$$
C_{R}=C_{e}=\frac{E_{2 f}-E_{1 f}}{E_{1 i}-E_{2 i}}
$$

Where: $C_{R}$ is the Coefficient of restitution, $C_{e}$ is the Coefficient of efficiency, $E_{1 i}$ is the initial energy of the first robot, $E_{2 i}$ is the initial energy of the second robot, $E_{1 f}$ is the final energy of the first robot, $E_{2 f}$ is the final energy of the second robot.

Since all robots are identical, they have similar energy losses, but as they reach different levels of energy the coefficient of restitution should be averaged.

Once robots are deployed, there could be two cases. The first case is when the robots' size compared to the distances travelled is negligible; this means that robots reaching preceding robots will have equal amounts of energy. The second case is when the robots' size is relatively large compared to the distance travelled (possibly due to harsh environments). This means that follower robots will have a slightly larger amount of energy than their preceding robot. When reaching saturation point (i.e. when the system is about to stop/fail) robots may be closely stacked behind one another with low energy. In this project robots stacked closely behind each other in that manner shall be called a 'slack-line'. Robots in a slack-line state are not expected to be able to move. If the team deploying the robots ensures that the slack-line occurs near the entrance, the whole group of robots forming the slack-line can be fully charged. An important question to ask is; what would happen if a robot was destroyed?

In order to understand the outcomes of how the different systems behave, a Matlab simulation has been created to find out each scenario's outcome.

\subsection{Matlab simulation:}

This simulation was constructed in order to answer the question - how many robots are needed to achieve what distance?

The answer differs according to robot design and terrain. To generate a generic model we simplify the problem by assuming that a unit distance is the distance equal to a robots length, Unit energy is the energy needed for a robot to move a unit distance. Robot's required travel distance is infinity. Robots consume the same amount of energy when crossing the same path.

\subsubsection{The first simulation:}

The first simulation tests the system without allowing slack-lines. It is expected to have robots stacked closely together just before the end of the simulation or saturation point. The following assumptions were taken:

- $\quad$ Robot's standby energy = 2 units

- A robot can move if it has more than 40 units 
- Each robot signals to the following robot once energy reaches $61 \%$

- At every simulated step robots loose 2 units of energy.

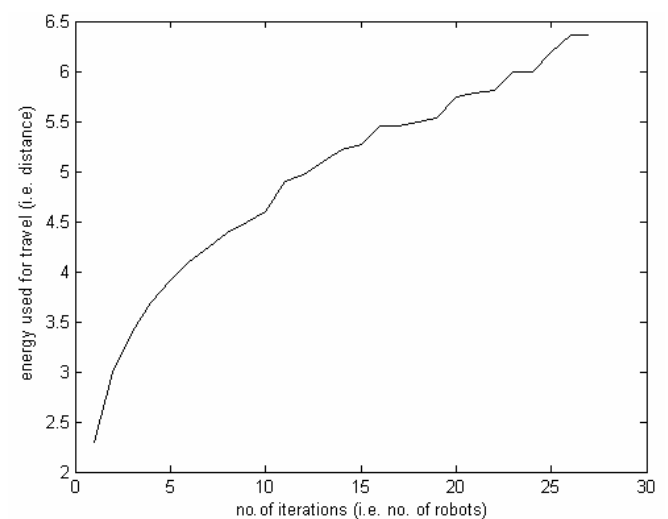

Figure 3.1 non-linear polynomial fit of no slack-line simulation output

- $\quad$ Coefficient of efficiency; $C_{e}=0.39$

Losing a robot mid operation will lead the system to an early fail/stop. How early, depends on which robot, and at what stage of the operation; see figure 3.1

\subsubsection{The second simulation:}

It is similar to the first simulation but when a slack-line occurs the teleoperator ensures that it starts at the entrance point. Robots forming the slackline can be fully charged to full capacity. Losing a robot in this case will dramatically affect the system and cause it to fail earlier, especially if the robot is part of the slack-line formation. See Figure (3.2).

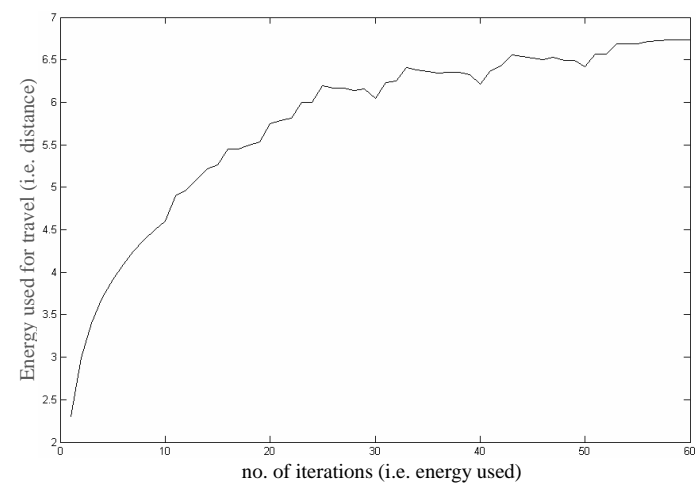

Figure 3.2 non-linear polynomial fit of slack-line simulation output 


\subsubsection{Practical experiment:}

This was done to study the effect of localisation inaccuracies; it can also give an idea of possible practical limitations. Due to the odometry inaccuracy, a robot's energy consumption for crossing the same area may be different to other robots. The following assumptions are made:

1. Each step costs the robot five units of energy,

2. Coefficient of efficiency $\left(C_{e}\right)=$ one.

3. Robots' standby energy $=2$ units.

4. Robots' can move if they have more than 25 units.

\subsubsection{The first practical experiment:}

In this experiment robots will signal to their followers at energy level 50 to prevent slack-line. Results were similar to the simulation. The leading robot's energy amount was 50 where the rest were at 25 . Regardless of inaccurate odometry, the experiment results were similar to that created by the simulation.

\subsubsection{The second practical simulation:}

In this experiment robots will signal to their followers at energy level of 61 to guarantee slack-line occurrence. Figure (3.3) shows an image of the formation of a slack-line, and another showing the system near the end of the experiment.

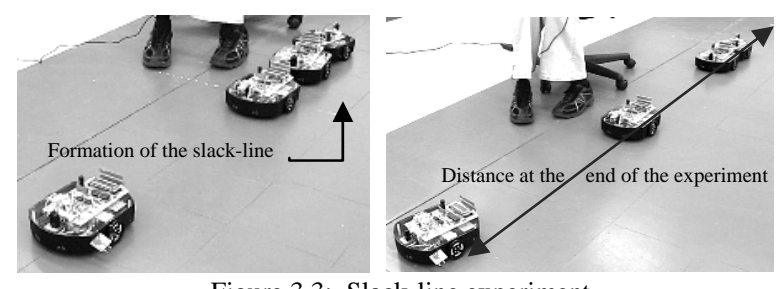

Figure 3.3: Slack-line experiment

Even with slightly inaccurate localisation, slack-line formation helped achieve greater distances. The experiment showed a similar pattern to the simulation.

\subsubsection{The third practical experiment:}

Here we assume that the travelled distances are much larger than the size of the robots, therefore each follower robot will reach its preceding robot with approximately the same energy. Distances were carefully measured to help manually re-adjust the robots' positions to counteract the knock on effect of the robots' size. Robots signaled to their followers when they reached an energy level of 65 units. 
The results obtained were similar to the ones obtained from the first experiment, but in this experiment a greater distance was covered. Both experiments indicated that at some point the system will come to halt.

\subsection{Results}

It is important to understand the output of experiment 3.2.3.

If a unit distance = distance achieved by using a whole battery.

If one robot was deployed only, then distance $=1$

If two robots were deployed, then maximum distance $=1.5$

If three robots were deployed, then maximum distance $=1.833$

The pattern generated follows the following equation (2):

$$
\text { Distance } \quad=\sum \frac{1}{n}
$$

The simulations in section 3.1.1and 3.1.2 show clear similarities. In section 3.1.1 the simulation has used fewer robots and achieved a shorter distance. The experiments have shown the same similarities. Experiment 3.2.2 has achieved greater distance than experiment 3.2.1. Regardless of changing of the constants and the odometry inaccuracy, the pattern has always been similar. The equations generated also show similar patterns. Looking into the distance achieved by the leading robot and comparing that to energy used and the number of robots a function that models the system can be generated. The distance can be calculated using the following equation (3):

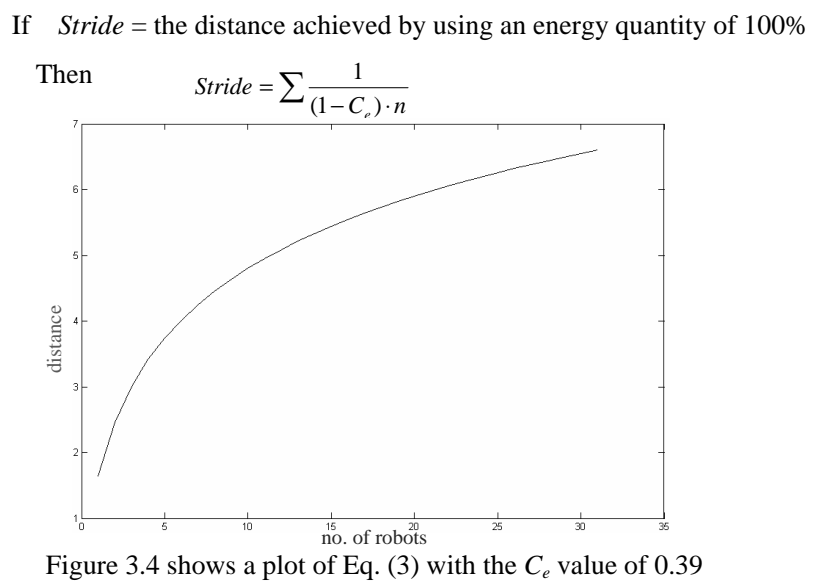

\section{Other methods:}

Looking at example (1) in section 3, a new case can be introduced, where the glass is passed without losses. For robots this means physically conveying 
the batteries. Robots should place an extra in the environment then head back to pick up another one. Once sufficient batteries are placed, robots can move deeper into the environment. Assuming that robots would drop the battery at a distance where they consume a third of their own battery; this enables us to plot what can happen in the long run. Because robots numerously go backwards and forwards, this method is classed as energy hungry. The pattern generated can help find a mathematical model. Figure 4.1 shows how that system behaves.

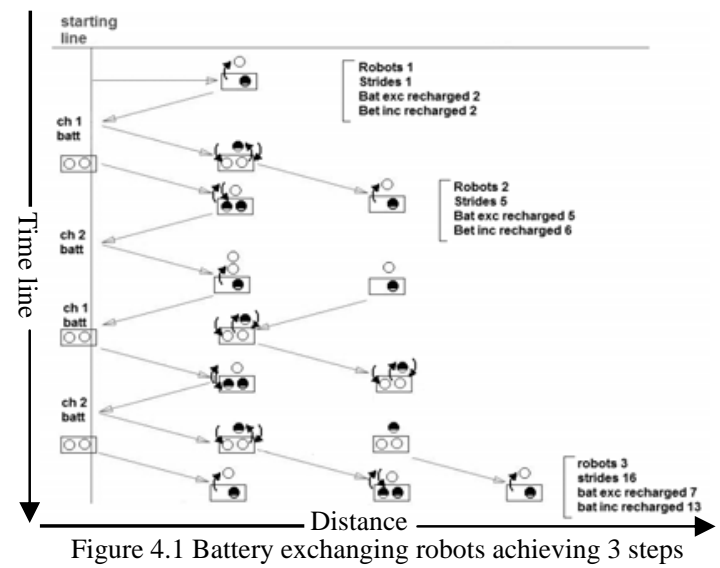

The energy used is measured by how many strides the system covers. Each stride costs a third of a battery. Each stage a robot goes deeper into the environment is classed a step. From Figure 4.1 the robots have covered three steps. A function has been generated for this system (see equation (4)). Note that if $S=1$ then Stride $=1 \&$ if $S=2$ then Stride $=5$.

if Stride = distance achieved by consuming $33 \%$ of one battery $\&$ if steps required $=S$ Then

$$
\text { if } S>2 \text { Then } \quad \text { Strides }=s+\sum_{1}^{S} 3^{(S-1)}
$$

\section{Conclusion:}

When designing a swarm of search and rescue robots with energy autonomy, the key factors are the coefficient of energy $\left(\mathrm{C}_{\mathrm{e}}\right)$ and how much energy the designer is willing to pay. Energy trophallaxis and energy storage is common in many areas in nature, particularly amongst ants. Myrmecocystus melliger ants (honey ants) are a good example of energy storage. An average rescue robot with batteries has the ability to go further than a hundred metres; even a third of that distance is about thirty three meters. If this system is going to be implemented, this could mean that robots will cover about a hundred to three hundred meters. This is highly sufficient for most search and rescue operations. If all aspects: communication, energy storage/exchange and energy 
autonomy are addressed, then a system of search and rescue robots that exchanges energy seams feasible.

\section{Future work}

Future work includes enabling the MARCO2 to pass a packet of first-response items and navigate through difficult terrain. More experiments are required regarding exchanging batteries. The expansion of the system has to be investigated, to allow robots to share energy in a tree formation. A user interface and dashboard must be created to control the semi-autonomous system and allow the robots to convey a map of the environment. Finally, a test arena must be established to help test the system more realistically.

\section{References}

1. Tsukagoshi H. et al (2005), 'Design of a Higher Jumping Rescue Robot with the Optimized Pneumatic Drive', Proceedings of the IEEE International Conference on Robotics and Automation, p. 1276 - 1283.

2. Burke J. L. , Murphy R. R. , Coovert M. D., Riddle D. L. (2004) 'Field Study of Human-Robot Interaction in the Context of an Urban Search and Rescue Disaster Response Training Exercise', Human-Computer Interaction, Volume 19, Issue 1 and 2, p. $85-116$.

3. Rybski, P.E.; Papanikolopoulos, N.P.; Stoeter, S.A.; Krantz, D.G.; Yesin, K.B.; Gini, M.; Voyles, R.; Hougen, D.F.; Nelson, B.; Erickson, M.D.(2000) 'Enlisting rangers and scouts for reconnaissance and surveillance', Robotics \& Automation Magazine, IEEE Volume 7, Issue 4, p. 14 - 24.

4. Basu, P.; Redi, J.(2004) 'Movement control algorithms for realization of faulttolerant ad hoc robot networks', Network IEEE, Volume 18, Issue 4, p. 36 - 44

5. Jiangyang Huang; S.M. Farritor; A. Qadi; S. Goddard. (2006) 'Localization and follow-the-leader control of a heterogeneous group of mobile robots', Mechatronics, IEEE/ASME Transactions on,Volume 11, Issue 2, p.205-215

6. Trung Dung Ngo; Henrik Schioler (2006), 'An Approach to Sociable Robots through Self-distributed Energy', IEEE/RSJ International Conference on Intelligent Robots and Systems, Volume 3, Issue 4, p. 2192 - 2199

7. Litus, Y.; Vaughan, R.T.; Zebrowski, P.( 10-14 April 2007),' The Frugal Feeding Problem: Energy-efficient, multi-robot, multi-place rendezvous', IEEE International Conference on Robotics and Automation, p. 27 - 32.

8. Melhuish C. \& Kubo M. (2004) 'collective energy distribution: maintaining the energy balance in distributed autonomous robots using trophallaxis’ Distributed Autonomous Robotic Systems 7, p. 261 - 270.

9. Dumpert, K (1981). The social Biology of Ants. Bath: The Pitman Press.102108. 